illustrated that use of BMS was independently associated with increased risk of MACE (HR 1.54; 95\% CI 1.05 to 2.25, $\mathrm{p}=0.03$ ), driven through an increase in revascularisation.

Conclusion In conclusion, in one of the largest analyses of its kind, use of DES in patients with diabetes in a real world setting undergoing PCI in large diameter coronary vessels $(\geq 3 \mathrm{~mm})$ is safe and is independently associated with a reduction in MACE events. This is in contrast to that of non-diabetic patients where the benefits of DES in large diameter coronary vessels are less evident.

\section{FALSE ACTIVATION FOR PRIMARY PERCUTANEOUS CORONARY INTERVENTION IS NOT A BENIGN PHENOMENON}

doi:10.1136/heartjnl-2011-300198.38

U Chaudhry, C Mavroudis, R D Rakhit. Cardiology Department, Royal Free Hospital, London, UK

Introduction Primary percutaneous coronary angioplasty (PPCI) is the preferred reperfusion strategy following an acute ST elevation myocardial infarction (STEMI). Since $200524 / 7$ primary PCI has been the first line treatment for an acute STEMI in our centre. 93\% of patients are direct access admissions by London Ambulance but a significant proportion (up to $20 \%$ ) do not fulfil the diagnostic criteria for STEMI and are termed "false activations". Data on the outcome of this cohort of patients is limited.

Aim To review the clinical outcome of patients presenting to our heart attack centre with false activation PPCI.

Method From January 2008 until October 2010, we identified 209 false PPCI activations defined as patients with incomplete diagnostic criteria for acute STEMI: absence of chest pain and/or typical ECG features (ST elevation or new LBBB). Data was collected via a "false activation" database together with retrospective review of case records.

Results Complete data was available in 165 cases. 71\% were male and $29 \%$ were female (mean age 67 ). The mean length of stay was 4 days (range $1-33$ ). $71 \%$ presented with chest pains and $29 \%$ had no chest pains, but presented with breathlessness, palpitations or syncope. The ECG abnormality was non-specific ST-T changes in $22 \%$, LBBB in $19 \%$, left ventricular hypertrophy in $15 \%$, fixed ST elevation or $\mathrm{Q}$ waves in $14 \%$, early repolarisation changes in $10 \%$, $\mathrm{RBBB}$ in $8 \%$ and other ECG abnormalities in 12\%. The final diagnosis was non-ST elevation acute coronary syndrome (NSTEACS) in $19 \%$, sepsis in $19 \%$ and congestive heart failure (CHF) in 15\%. Stable angina was observed in $8 \%$ and syncope in $7 \%$. Musculoskeletal or non-cardiac chest pains were noted in $8 \%$ and $7 \%$ of the patients respectively. $2 \%$ of the patients had pulmonary embolism and in $5 \%$, a gastric cause for presentation was diagnosed. 14\% had other cardiac problems, including arrhythmia, dilated cardiomyopathy, hypertension, pericarditis, pericardial effusion and late presentation STEMI. $15 \%$ had other diagnoses. The mean follow-up period was 18.7 months, during which $21.5 \%$ of false PPCI activation admissions died $(n=45) .25 \%(n=11)$ died during the index admission and $33 \%(n=15)$ died within 30 days of admission. The overall 30-day mortality for false activations was $7.2 \%$, which is higher than the overall PPCI mortality of $6.0 \%$ (including cardiogenic shock) $(p=0.008)$ and $3.3 \%$ (excluding shock) $(p<0.0001)$ in our centre. $49 \%$ of deaths were cardiac (NSTEACS and CHF), 29\% sepsis and $22 \%$ other causes. The mean age for this cohort was 83.

Conclusion Patients presenting with false PPCI activation have a high observed mortality. This is probably due to significant associated comorbidities, including occult cardiac disease. Thus, false PPCI activation is not a benign phenomenon and masks underlying significant disease. Robust pathways are required to minimise delay in further investigations and a need for risk stratification for a significant proportion who present with NSTEACS.

\section{A RANDOMISED CONTROLLED TRIAL COMPARING CONVENTIONAL CORONARY ARTERY BYPASS GRAFT SURGERY WITH A COMPOSITE ARTERIAL GRAFT TECHNIQUE}

doi:10.1136/heartjnl-2011-300198.39

${ }^{1}$ A Alahmar, ${ }^{2} \mathrm{R}$ A Perry, ${ }^{2} \mathrm{R}$ H Stables. ${ }^{1}$ Leicester University Hospital Glenfield, Leicester, UK; ${ }^{2}$ Liverpool Heart and Chest Hospital, Liverpool, UK

Background Composite (Y/T) coronary artery bypass graft surgery (CABG) confers full arterial revascularisation, and "hands off" aorta compared to conventional bypass graft surgery. However, the composite surgical configuration could lead to preferential blood flow down one arm than the other (left internal mammary artery LIMA or radial artery RA) with its potential impact on graft patency.

Aim To investigate the impact of bypass graft configuration on short-term grafts patency and cardiac related quality of life.

Methods and Results This is a single centre randomised, controlled trial Between March 2006 and July 2007, 322 patients undergoing isolated bypass graft surgery at our institution were screened and $89(27 \%)$ met the inclusion criteria and were randomised. Patients were allocated to conventional (conv $\mathrm{n}=46$ ) or composite (comp $\mathrm{n}=43$ ). The two primary end points were graft patency defined as (Thrombolysis In Myocardial Infarction) TIMI III flow in distal anastomosis at angiography 12-24 months after surgery, and cardiac-related health status assesses by Seattle angina questionnaire (SAQ). Baseline characteristics were similar between the two groups apart from diabetes where there were more diabetic patients in the composite arm than the conventional one $(15(35 \%)$ vs $5(11 \%)$ p $<0.01$ respectively). Trial was stopped prematurely following 18 months interim analysis which showed significant graft failure in the composite arm (40\%). Final Analysis was performed on intention to treat basis. Sixty-five (73\%) had follow-up angiography (34 conv, 31 comp), with total of 116 graft in conventional arm and 100 grafts in composite arm. All patients in both groups had LIMA graft to left anterior descending artery (LAD). Graft patency rate was significantly higher in the conventional compared to composite $\operatorname{arm}(95(82 \%)$ vs $59(59 \%) \mathrm{p}<0.001$ respectively). Three main domains of the SAOs there was significant improvement between before and 6 months after surgery in both groups. There were no significant differences between the two groups in the percentage of improvement in these four domains (Physical limitation, Angina stability, Angina frequency, Quality of life).

Conclusions In our randomised trial, composite bypass graft surgery was associated with higher graft failure rate at 12-24 months after surgery compared to conventional type. This difference may be due to the composite conduit configuration. Further blood flow characteristics study in this configuration can help understand such an important finding and its implication on our clinical practice. Despite the difference in graft patency there were no differences in physical limitation, angina stability, angina frequency, or quality of life between the two groups.

\section{PATIENT VS PHYSICIAN REPORTED ANGINA BEFORE AND AFTER REVASCULARISATION OF CORONARY ARTERY DISEASE: EVIDENCE FROM A LARGE RANDOMISED CONTROLLED TRIAL (THE SOS TRIAL)}

doi:10.1136/heartjnl-2011-300198.40

C Appleby, I Kemp, R H Stables. Liverpool Heart and Chest Hospital, Liverpool, UK

Introduction The success of revascularisation therapies for coronary artery disease (CAD) must be measured by both an improvement in hard clinical endpoints-mortality, repeat revascularisation procedures and myocardial infarction, the traditional focus of clinical trials-and, critically for patients, the relief of angina symptoms. Interest in patient reported outcomes (PROMs) has increased, although their use in cardiovascular trials is far from universal. In 